\title{
Measurements of reference ISO nozzles by high-speed imaging
}

\author{
Nicolas De Cock ${ }^{\mathrm{a}, \mathrm{b}, *}$, Mathieu Massinon ${ }^{\mathrm{a}}$, David Nuyttens ${ }^{\mathrm{c}}$, Donald Dekeyser ${ }^{\mathrm{c}}$, \\ Frédéric Lebeau ${ }^{\mathrm{a}}$ \\ ${ }^{a}$ University of Liege, Gembloux Agro-Bio Tech, Passage des déportés 2, 5030 Gembloux, \\ Belgium \\ ${ }^{b}$ von Karman Institute, Environmental and Applied Fluid Dynamics Department, 1640 \\ Rhode-Saint-Genèse, Belgium \\ ${ }^{c}$ The Institute for Agricultural and Fisheries Research (ILVO), Technology and Food \\ Science Unit, 9820 Merelbeke, Belgium
}

\begin{abstract}
Agricultural spray characteristics determine the efficiency of a pesticide application because size and velocity affect droplet trajectory and impact behavior. At present, the relevance of different characterization techniques remains controversial since discrepancies may be significant between measurements performed in different laboratories.

A digital image acquisition technique and analysis algorithm is proposed for droplet size and velocimetry measurements as an alternative to well-established techniques such as the Phase Doppler Particle Analyzer (PDPA) or laser diffraction spectrometry (LDS). The algorithm requires double exposed shadow images acquired in a back-lighted arrangement with a Particle Image Velocimetry (PIV) camera and a pulsed light emitting diode (LED). Spatial illumination heterogeneities are corrected by subtracting from each image a mean background acquired on several images without any particle. The algorithm accuracy is ensured by the rejection of out-of-focus particles using a focus parameter depending on gradient intensity at the particle edges. Thresholds for focus particle selection were determined by studying the evolution of the focus parameter and the error on particle size measurements from images containing droplets with
\end{abstract}

\footnotetext{
${ }^{*}$ Corresponding author

Email address: nicolas.decock@ulg.ac.be (Nicolas De Cock)
}

Preprint submitted to Journal of Crop Protection

July 12, 2016 
uniform size at various distance of the object plane. Selected droplets were identified on both pairs of images to determine their size and velocity. Droplet size distributions were corrected to account for the uneven sampling probability caused by the volumetric method.

Droplet size distributions of a set of reference nozzle/pressure combinations defined in the ISO/DIS 25358 were measured. The image technique was able to distinguish each of the reference sprays well. Comparison with PDPA measurements showed that the imaging technique tends to measure an equivalent $D v_{50}$, a lower $D v_{10}$ and a higher $D v_{90}$ leading therefore to a higher relative span factor. Velocity measurements showed good agreement between both techniques except for one nozzle/pressure combination.

Keywords: Droplet sizing, digital image analysis, agricultural spray, ISO/DIS 25358

\section{Introduction}

Droplet size and velocity distributions determine the overall treatment efficiency as they influence two specific steps of the pesticide application process, namely deposition and retention (Zabkiewicz, 2007). Deposition corresponds

5 to the droplet transport from the nozzle to the target (weeds, insects, plant pathogens, etc.) or the amount of pesticide directed within the target area. Deposition efficiency is then defined as the ratio between the volume of droplet that reaches the target and the total volume sprayed. Deposition is optimized when the probability of a droplet to collide with the target is maximal considering the whole droplet size and velocity distributions. Physical transport of droplets (Wang et al., 1995: Walklate, 1987) and spray drift potential (Holterman et al., 1997, Lebeau et al., 2011, Teske et al., 2002) have been investigated and modeled intensively based on spray characteristics (droplet size and velocity distributions) and environmental conditions (release height, meteorological conditions, etc.) to improve deposition. Retention is the part of the deposited volume effectively retained by the plant. Its efficiency is determined by the 
contribution of each spray droplet during impact on the target (Massinon et al. 2015). The impact behavior depends on droplet and surface properties (Rein, 1993). Flying insect control, such as mosquitoes, requires small droplets $(\leq 50$ ${ }_{20} \mu \mathrm{m}$ ) for maximizing retention but are, however, airborne for a longer time than large droplets. Herbicide treatment usually involves larger droplets (200-300 $\mu m)$ which are less sensitive to drift than small droplets but leading to droplet rebound and fragmentation during impact on the target. Nozzle classification according to droplet spectrum is an indicator for the most appropriated treatment for a given product and target.

The first nozzle classification was developed by the British Crop Protection Council (BCPC) in 1985. Droplet size distributions of test nozzles are compared to those of a set of reference nozzles which delimit the midpoint between five size classes, from very fine to very coarse. The classification was improved to 30 include spray drift potential and reference classification curves were changed from midpoints to thresholds (Southcombe et al., 1998). The American Society of Agricultural Engineers (ASAE) further expanded this classification with an additional extra-coarse class for anti-drift nozzles (Hewitt et al., 1998). Interlaboratory (round-robin) evaluations are often performed using the same set of reference nozzles to compare spray quality classification between methods (Fritz et al. 2012) and to account to the weak uniformity in the manufacturing of commercial nozzles (Womac, 2000). These measurements showed considerable differences between methods.

Aside agriculture, measurement of particle size and velocity is common in various domains including fire safety (Widmann, 2001, Zhou et al., 2012), pharmaceutical delivery (Liu et al., 2010), engine technology (Li et al., 2011), geomorphology (Kang et al. 2008), painting (Snyder et al., 1989) and food technology (Kwak et al. 2009). This resulted in many measurement methods with different advantages and drawbacks. Most of the non-intrusive techniques are 45 optic based, i.e., Phase Doppler Particle Analyser (PDPA), Laser Diffraction Spectrometry (LDS), imaging techniques (Shadowgraphy, PDIA). PDPA measures particle size and velocity from the light scattered by a particle moving 
through a measurement volume, which is defined by the interference of two focused laser beams. PDPA measurement requires liquid optical properties (refractive index) and is limited to spherical particles (Damaschke et al. 1998). LDS measures the diffraction pattern formed by the particles inside the probe volume. Droplet size distribution is found by using the complete Mie theory or the Fraunhofer approximation of the Mie theory on the recorded diffraction pattern (ISO 13320:2009). This method provides spatial measurement of particle size distribution without information on particle velocities. PDPA and diffraction methods require coherent light source from laser and dedicated electronics and optics, which induce a high cost.

Particle/Droplet Image Analysis (PDIA), usually performed in back-lighted arrangement is often referred as shadowgraphy. Particles that are significantly bigger than the light wavelength located in the probe volume, which is defined by the camera field of view and the depth of field, intercept the light and cast their shadows on the camera sensor. Particle size and centroid coordinates are determined by digital analysis of these shadows. Velocity measurement requires a tracking algorithm that identifies the same particle on two successive frames. rangement offers relatively low influence of particle shape and liquid optical properties on particle size and velocity measurement (Lecuona et al. 2000) and requires no delicate optic alignment. Accuracy of the particles size measurement is determined by the device's ability to correctly identify particle edges. its are easily established using the higher intensity gradient on image. Because of out-of-focus phenomena and motion blur, the contrast may be lower, inducing uncertainties and errors on particle size measurement. Motion blur can be avoided by adjusting exposure time or light pulse length depending on particle 75 velocities. Out-of-focus effect is dealt using a parameter that expresses the focus degree of the particle according to two main approaches: the ratio between intensity gradient on particle boundary and the contrast between particle and background (Lecuona et al. 2000), or the area of the gray halo around particle 
shadow (Kashdan et al. 2003). Based on this parameter, the out-of-focus particles can be rejected by thresholding. The suited threshold level is chosen after determining size and focus level of known size particle at different positions around the object plane by calibration. The threshold level determines also the depth of field of the measurement volume, which is defined by the distance along the optical axis over which the uncertainty results in an acceptable error on the measured diameter. Depth of field grows typically with the particle size (Kashdan et al., 2003).

The rapid development of imaging equipment during the last decade makes shadowgraphy an even easier to use and a cheaper alternative to scatter or diffraction based measurement methods for micro-metric particles. A digital 90 Particle Image Velocimetry (PIV) camera combined with standard optics and pulsed Light Emitting Diodes (LED) arrays as light source provide a relatively low cost acquisition system. This multipurpose equipment can also be used for qualitative observations such as liquid sheet break-up (Cousin et al., 2012) or agricultural spray impact retention (Massinon and Lebeau, 2012; Massinon

et al., 2014), what results in a very versatile tool for laboratories involved in spray application processes.

The aim of this paper is to gather recent developments in shadow image processing needed to develop an accurate, versatile and low-cost tool to characterize agricultural spray quality. The technique was evaluated with a high-speed PIV 100 camera combined with a pulsed LED array back-light source. The developed tool was assessed by characterizing the droplet size distribution of the 6 spray quality boundaries defined by in ISO/DIS 25358 standard for the classification of droplet size spectra from atomizers. The results obtained with the imaging technique were compared with PDPA measurements. 


\section{Materials and methods}

\subsection{Experimental set-up}

Shadowgraphy involved a back-lighted arrangement for image acquisition (Figure 1). A PIV camera (X-Stream XS-3, Integrated Design Tools, Tallahassee, FL, USA) coupled with high magnification optics provides a field of view of $10 \mathrm{~mm} \times 12 \mathrm{~mm}$ at a working distance of $130 \mathrm{~mm}$. The spatial resolution is equal to $9.7 \mu \mathrm{m} /$ pixel. With this magnification factor, droplets with a diameter ranging from 40 to $3500 \mu \mathrm{m}$ can be measured. A custom made $72 \mathrm{~W}$ LED array (24 Luxeon III Star White) was placed $500 \mathrm{~mm}$ from the camera. A LED-controller (PP600F, Gardasoft Vision, Cambridge, UK) provided repeatable intensity control of the LED lighting. Possible shortest pulse length provided by the illumination system was $1 \mu s$ and was triggered by the image acquisition. Digital images were 1024 x 1280 matrices in which each value is the light intensity recorded by a camera pixel. In order to avoid motion blur a short exposure time was used $(3 \mu s)$. Using the double exposure mode of the PIV camera, two consecutive images were acquired within a short time (38 $\mu s)$ allowing the computation of the droplet displacements and subsequently the droplet velocities. The probe volume of this technique corresponds to the volume in which the droplets appear sharp enough to be measured with an acceptable error $(\leq 5 \%)$. A droplet has to appear in both frames of a pair of images to be taking in account. Hence the size of the probe volume is decreasing with the droplet speed. Calibrations showed that this volume is a rectangular parallelepiped with a maximum size of $10 \times 12 \times 1 \mathrm{~mm}^{3}$.

\subsection{Image processing}

Figure 2 presents the main steps for image processing starting from the raw image. In the first step, raw image quality is improved by background subtraction (2.2.1). As the background changes with optics alignment and camera settings, the background images have to be taken with the exact same set-up. In a second step, the image segmentation is performed in two phases. 


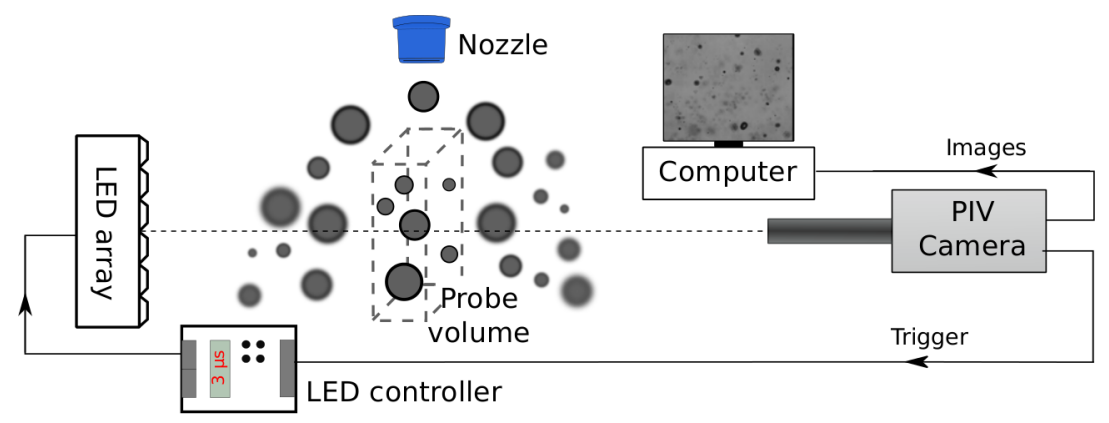

Figure 1: Shadowgraphy set-up used for the image acquisitions.

\begin{tabular}{|c|c|c|c|}
\hline Process & \multicolumn{3}{|c|}{ Illustration } \\
\hline Raw image & $\ddots$ & & \\
\hline $\begin{array}{c}\text { Background } \\
\text { substraction }\end{array}$ & $\ddots$ & & \\
\hline $\begin{array}{c}\text { Droplet } \\
\text { localization }\end{array}$ & & & \\
\hline $\begin{array}{c}\text { Droplet } \\
\text { sizing }\end{array}$ & 0 & 0 & \\
\hline $\begin{array}{c}\text { Out of focus } \\
\text { droplet rejection }\end{array}$ & $\ddots$ & & \\
\hline
\end{tabular}

Figure 2: Main steps of the droplet sizing algorithm illustrated with the example of the selection of droplets starting from a raw image.

Firstly, the droplets present on the images are identified and isolated in subimages (2.2.2). Secondly, each droplet is individually segmented from the local background using by the Canny edge detection algorithm (Canny, 1986) (\$2.2.3. Finally, the out-of-focus particles are rejected on the basis of a focus parameter in order to ensure an accurate sizing (\$2.2.4.

\subsubsection{Background subtraction}

Correction for the spatial illumination heterogeneity consists of subtracting the background from each image. A composite background is then generated from the 80 percentile of each pixel intensity on a set of 50 images. Finally, after the background subtraction the image gray level is rescaled in a way that 
$1 \%$ of pixels are saturated (i.e. equal to 0 or 255 ) to maximize image contrast,

\subsubsection{Droplet localization}

The droplet shadows present a variable gray level depending on the droplet size, degree of focus and local illumination. Consequently, there is no unique threshold adapted for an accurate segmentation of all droplets. Therefore, each droplet is analyzed individually in order to take into account the local image context. The first localization step of the droplets is achieved by computing the light intensity gradient on the whole image using Sobel's filters. The highest intensity gradients generally correspond to object boundaries. Therefore, the chosen threshold should be sufficiently low to detect all droplet boundaries, computing the inner area defined by the edge.

\subsubsection{Out-of-focus droplet rejection}

Rejection of out-of-focus particles is essential for an accurate particle sizing. Droplet degree of focus is related to the distance between the particle and the focal plane. Selection of particles with a minimal degree of focus determines the depth of field measurement and, consequently, the sampling volume. A wellfocused object exhibits a sharp transition with the background at its boundaries, 
while the degree of focus decreases as a droplet moves away from the focal plan and a larger gray halo appears around the object. Gradient intensity at particle boundaries increases with particle degree of focus. Based on this observation, a focus parameter adapted from the in-focus parameter of (Lecuona et al., 2000) is proposed:

$$
\text { Focus parameter }=\frac{\text { grad }_{\text {bound }}}{I_{\text {object }}-I_{\text {back }}}
$$

where $\operatorname{grad}_{\text {bound }}$ is the intensity gradient value on the object boundaries, $I_{\text {object }}$ and $I_{b a c k}$ are gray levels of the object and the background, respectively. To avoid effect of noise or the bright spot caused by light scattering, these last values are obtained with a rank order filter such as median value. This focus parameter is less sensitive to local illumination variations since it is based on the contrast between the object and the local background. Thresholds for focused particle selection were determined by studying the evolution of the focus parameter and the error on particle size measurement from images containing uniform droplets with a known size at various distance of the object plane. This was achieved using a custom-made droplet generator that produces a continuous stream of equally spaced and mono-sized droplets. The generator produce a round jet which is broken into droplets by stimulating the Plateau-Rayleigh instability at an optimal frequency by mechanical vibrations (Sirignano and Mehring, 2000). Five glass nozzles producing droplets of 111, 157, 208, 351 and $516 \mu m$ were used. The droplet diameters at optimal perturbation frequency were calculated by the following equation:

$$
d=\sqrt[3]{\frac{6 Q}{\pi f}}
$$

where $d$ is the droplet diameter $[m], Q$ is the flow rate measured by bucket method $\left[\mathrm{m}^{3} / \mathrm{s}\right]$ and $f$ is the perturbation frequency $[\mathrm{Hz}]$. The stream of droplets was shoot with an oblique direction in respect to the focal plane. Examples of oblique droplet streams are presented in the Figure 3 a. By recording around hundred pictures, a continuous expression of the focus parameter and the relative error on the droplet size measurements could be expressed in respect to the focal plane distance (Figures $3 \mathrm{~b}$ and c). Thresholds of the focus parameter 


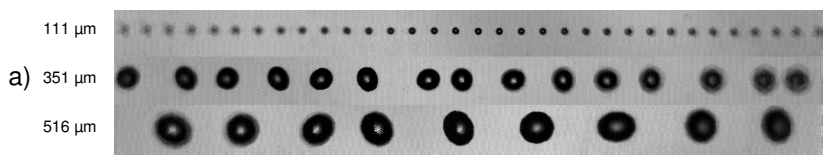

b)

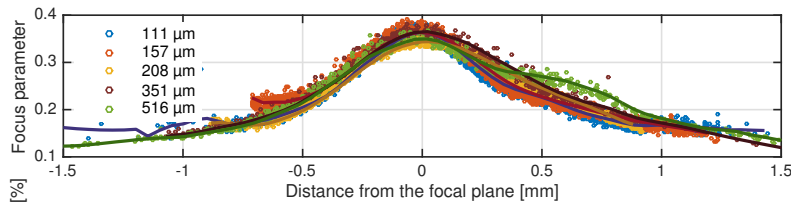

c)

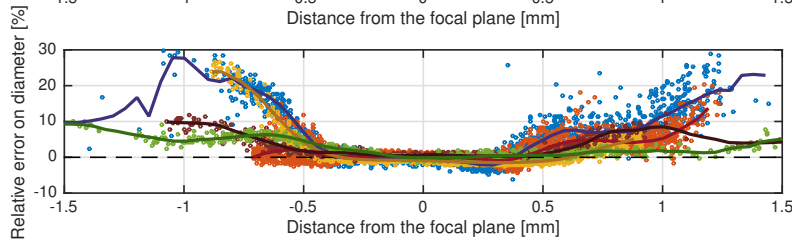

Figure 3: Oblique shoot of 111, 351 and $516 \mu \mathrm{m}$ droplet streams (a). Focus parameter (b) and relative error on the diameter measurement (c) in respect to the distance from the focal plane.

were then chosen to have both an error less than $5 \%$ on size measurement and and a depth of field as large as possible. Figure 4 shows the relation between the depth of field and the droplet diameter. A threshold of the focus parameter equal to 0.23 was chosen giving the following linear relationship between the depth of field (DOF) and the diameter (d) (both expressed in $m$ ):

$$
D O F=0.85 d+0.00078
$$

\subsection{Particle tracking velocimetry (PTV)}

Particles are tracked between image pairs for velocity computing. Several criteria are required to find the same droplet on two successive frames with a high level of confidence. The most evident criterion is the conservation of diameter. The second is the displacement expected between two frames. In an agricultural spray the mean droplet direction is known, providing a hypothetic localization of a droplet on the second exposure. The search area on the second frame was defined as a circular sector oriented along the mean flow direction (Figure 5). 


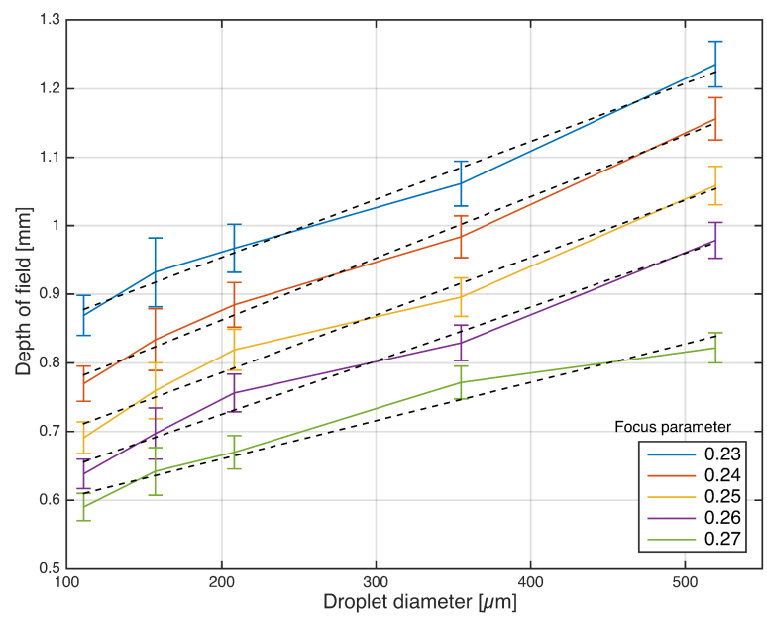

Figure 4: Depth of field for the different droplet diameters according to the focus parameter. The dashed lines correspond to linear regressions.

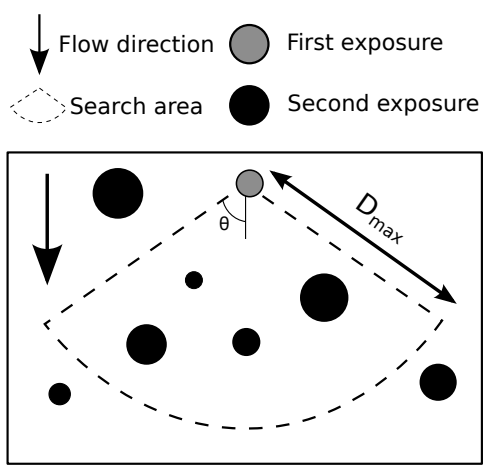

Figure 5: Droplet tracking principle using a search area based on the size conservation of the droplet and a priori knowledge of the flow direction in order to retrieve the same droplet on two successive frames. 
The opening angle $\theta$ is defined by the maximum angle between the main flow direction and a particle displacement, depending on radial dispersion intensity. Maximal displacement of a particle between two frames is determined according to the delay between the two exposures and a maximal velocity assumption for the spray:

$$
D_{\max }=v_{\max } \Delta t
$$

where $D_{\max }$ is the maximal displacement $[\mathrm{m}], v_{\max }$ is the maximal velocity $[\mathrm{m} / \mathrm{s}]$ and $\Delta t$ is the time between the two exposures $[\mathrm{s}]$. The values chosen for the displacement and the distance criteria define the measurement confidence. Too permissive criteria provide mismatching and finally error in computed velocity. Inversely, too restrictive criteria limit the pair matching and result in a mislead-

225 ing velocity measurement. These errors can be removed by post processing or by adjusting the maximum displacement to the droplet diameter according to an iterative procedure thanks to the high velocity-size correlation into sprays (Lefebvre, 1988).

\subsection{Droplet size distribution}

Droplets do not have an equal probability to be measured due to the volumetric sampling method. Sampling probability is depending both on the size of the probe volume and on the residence time of the droplets into this volume, which depends on droplet velocity and size. A slow droplet remains longer in the probe volume and in turn is more likely to be recorded on the subsequent frame than a fast droplet.

Furthermore, the larger the droplet, the higher the probability to touch the image edges and to be rejected during the object localisation step. Droplet size distribution is established by weighting the volumetric contribution of the accepted droplets by a correcting factor $(C F)$, which is defined as follows:

$$
C F=\frac{v}{F O V_{c o r} D O F}
$$

where $v$ is the droplet velocity $[\mathrm{m} / \mathrm{s}], D O F$ is the optical set-up depth of field $[m]$ expressed by equation 3 in respect to the droplet diameter and $F O V_{c o r}$ is the 


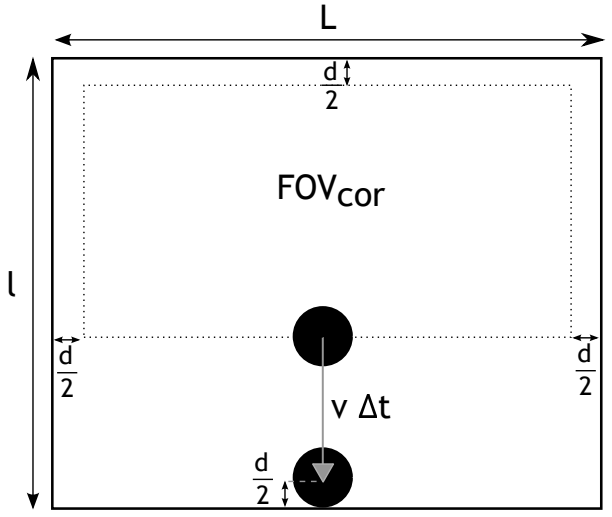

Figure 6: Illustration of the corrected field of view $\left(F O V_{c o r}\right)$ in dashed line which is defined as the area on the first image wherein the droplet center must be located in order to be measured. To be measured a droplet cannot be cropped by the image edge and has to fully appear on the second image.

corrected camera field of view $\left[\mathrm{m}^{2}\right]$, which is the image area in which a droplet must occur in the first acquisition to be measured (Figure 6) and is determined as follows:

$$
F O V_{c o r}=[(l-d)-(v \Delta t)](L-d)
$$

where $L$ and $l$ are the length and the width of the image respectively $[m], d$ is the droplet diameter $[m]$ and $\Delta t$ is the time between two exposures $[s]$. It is assumed that the vertical component of the droplet velocity is much larger than the horizontal one, which means that the algorithm does not take into account the possible exit of a droplet from the side of the image.

\subsection{Image processing implementation}

Matlab R2013a with image processing toolbox was chosen as technical computing language to implement the above image processing and analysis. The Matlab routines are available with an example at the permanent URL: http: //hdl.handle.net/2268/150929 
Table 1: Combination of nozzle and pressure defining the different spray class boundaries with the respective nominal flow rate.

\begin{tabular}{c|c|c|c}
$\begin{array}{c}\text { Spray class } \\
\text { boundary }\end{array}$ & Nozzle & Pressure [bar] & $\begin{array}{c}\text { Nominal } \\
\text { flow rate [L/min] }\end{array}$ \\
\hline \hline VF/F & Teejet TP 110 01 & 4.5 & 0.48 \\
F/M & Teejet TP 110 03 & 3 & 1.18 \\
M/C & Teejet TP 110 06 & 2.5 & 2.16 \\
C/VC & Teejet TP 80 08 & 2.5 & 2.88 \\
VC/UC & Teejet TP 65 10 & 1.5 & 2.80 \\
UC/XC & Teejet TP 65 15 & 1.5 & 4.18
\end{tabular}

\subsection{Reference nozzles-pressure combinations}

Spray characterization was performed on a set of reference nozzles using the imaging technique and compared with the PDPA laser technique. Six stainless steel flat fan nozzles (Sprayings Systems Co., Wheaton, USA) are currently used in round-robin tests in the framework of the ISO/DIS 25358 " Crop protection equipment - Droplet-size spectra from atomizers - Measurement and classification", to define boundaries for nozzle classification: Very Fine (VF), Fine (F), Medium (M), Coarse (C), Very Coarse (VC), Ultra Coarse (UC) and Extremely Coarse $(\mathrm{XC})$. The six nozzle/pressure combinations are presented in Table 1 Tap water was used as liquid and the spray pressure was set with a maximum relative error of $3 \%$.

\subsection{Measuring protocol}

\subsubsection{Imaging technique}

For the imaging technique, the measurements were realized $0.5 \mathrm{~m}$ below the nozzle and covered $1 / 4^{\text {th }}$ of the whole spray assuming the spray to be symmetrical (Figure 7). The scan of the spray was realized by recordings 1500 pair of images per line on 8 lines of $0.85 \mathrm{~m}$ spaced by $0.001 \mathrm{~m}$. During the recording of the images, the nozzle was moving at $0.0425 \mathrm{~m} / \mathrm{s}$ along the spray major axis. 


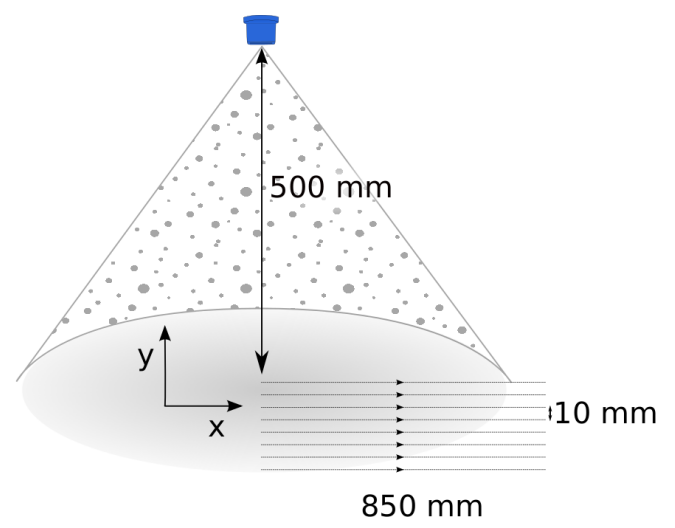

Figure 7: Scanning pattern used for the characterization of the sprays.

Finally, the droplet size distribution was retrieved by gathering the data from each scanning line. For the coarser nozzle-pressure combination (UC/XC), 2250 pairs of images per line have been taken in order to have sufficient amount of droplets.

\subsection{2. $P D P A$}

A TSI/Aerometrics PowerSight solid state laser-based PDPA system was used (Nuyttens et al., 2007). The system comprises an Argon-Ion laser, a fiberoptic transmitter and receiver, a signal analyzer, and FlowSizer-software. By means of the fiber-optic transmitter the laser beams are focused to cross over at a distance equal to the focal length $(500 \mathrm{~mm})$ of the transmitter lens. The sampling area is formed by the intersecting beams and has the shape of an ellipsoid. When a droplet passes the sampling area, the laser light is refracted. The fiber-optic receiver collects the scattered laser light. The light is directed by a prism pack to three photomultiplier tubes (PMTs) which convert the light signals into electrical signals to be processed for velocity and size information by the signal analyzer. Each PMT produces a signal with a frequency proportional to the particle velocity taken perpendicularly to the sampling area. Therefore, the measured velocity corresponds to the vertical component of the droplet velocity. The phase shift between the signals from two different PMTs 
is proportional to the size of the spherical particles. Measurement ranges for velocity and diameter can be changed through variations in the optical equipment, laser beam separation, and lens focal lengths of the transmitting and receiving optics. Settings on the instrument were chosen to cover a size range of 3 to 1113 $\mu m$. A cross-section average sample was obtained across the spray plume at $0.5 \mathrm{~m}$ distances from the nozzle outlet by moving the nozzle on a scan pattern. For the PDPA measurements, the full spray pattern was sampled by scanning 9 lines. In general, a different scan trajectory was programmed depending on the type of nozzle. Scanning speed was set that each scan yielded data for at least 10000 droplets with the PDPA.

\section{Results and discussion}

\subsection{Droplet measurement}

The post processing of the 12000 pairs of images per nozzle-pressure combination took in average 100 minutes. Matlab R2013a was used on a desktop computer with a processor Intel i7-4930k and 16 go of ram. The table 2 presents the number of droplets for each nozzle-pressure combinations. For the imaging technique, 15000 to 95000 droplets were recorded at the end of the whole scan. In order to have more than 15000 droplets, extra videos have been recorded for the UC/XC spray. For the PDPA, from 50000 up to 85000 droplets were measured during the scanning process. The rejected droplets for the imaging correspond to the droplets detected on the first frame which couldn't be track on the second frame. The rejected droplets for the PDPA are due to three reasons. Firstly, there is a size-intensity validation, a certain droplet size should have a certain range of intensity of the refracted light. Secondly, extreme values are rejected by putting some ranges (this is rejecting only 1 or a few droplets per scan). Thirdly, only droplets where we have values for size and velocity are kept. PDPA presents lower amount of rejected droplets, however rejection arise from different origin so it's difficult to compare. 
Table 2: Number of droplets measured and rejected with both technique for each nozzlepressure combinations.

\begin{tabular}{c||c|c||c|c}
\multicolumn{1}{c||}{} & \multicolumn{3}{c||}{$\begin{array}{c}\text { Number of } \\
\text { measured droplets }\end{array}$} & \multicolumn{2}{c}{ rejected droplets [\%] } \\
\cline { 2 - 5 } & Imaging & PDPA & Imaging & PDPA \\
\hline \hline VF/F & 96918 & 75205 & 10.2 & 5.1 \\
F/M & 47322 & 79191 & 9.1 & 2.9 \\
M/C & 40720 & 77909 & 9.2 & 3.8 \\
C/VC & 35573 & 85630 & 14.9 & 4.5 \\
VC/UC & 15998 & 69821 & 9.0 & 6.8 \\
UC/XC & 20682 & 55095 & 8.5 & 4.1
\end{tabular}

The spatial distribution of the accepted and the rejected droplets for the imaging technique can be displayed over the field of view. The figure 8 aggregated the accepted and the rejected droplets from all the nozzle-pressure combinations measurements. Each pixel of the figure corresponds to a square of $500 \mu \mathrm{m} \times 500 \mu \mathrm{m}$ on the field of view. The accepted droplets are quite uniformly distributed, except on the sides of the field of view were less droplets have been measured because in this area the droplets are more prone to exit the field of view on the second frame or to be cropped by the image border. A large volume of the rejected droplets $(65 \%)$ is located in the lower $1 \mathrm{~mm}$ of the field of view. Since the droplets are crossing the field of view from the top to the bottom, this high rejection rate is due to the exit of the droplet from the field of view between the first and the second frame. The droplet rejected on the sides of the field of view $(1 \mathrm{~mm}$ from the side and neglecting the lower 1 $\mathrm{mm}$ ) represent $9.4 \%$ of the overall rejected volume. There is a higher droplet rejection $(5.4 \%)$ on the right side of the field view than on the left side $(4 \%)$. This dissymmetry is explained by the fact that only the right half side of the spray triangle is scanned. Therefore, it corresponds to the droplet on the edge of the spray which have a higher horizontal velocity. The exit of the field of 

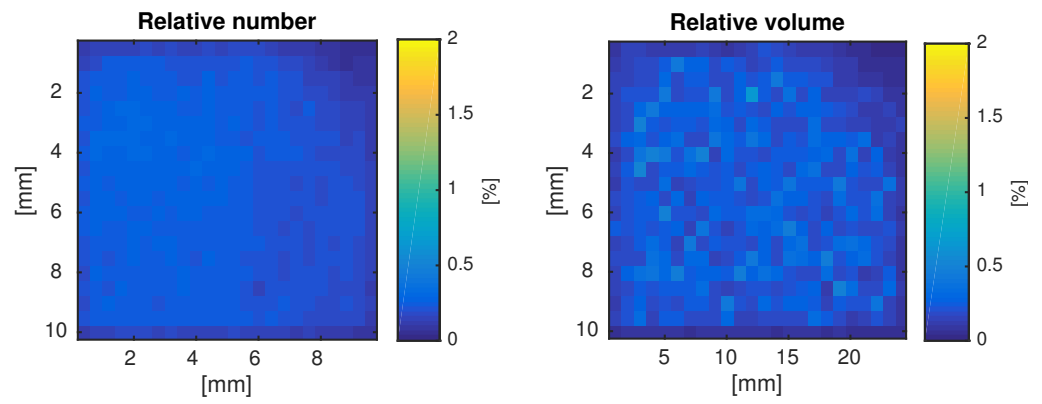

(a) Accepted droplets
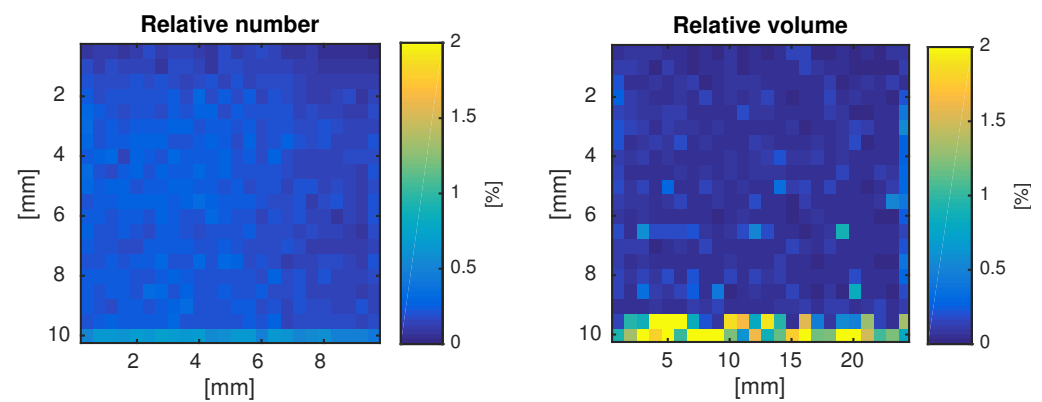

(b) Rejected droplets

Figure 8: Spatial distribution of the rejected and the accepted droplets on the field of view. The droplets from all the imaging measurements have been used to build this spatial distribution.

view by the droplets are mainly occurring at the bottom side as supposed by the field of view correction which has been proposed in 2.4. The other rejected droplets may come from droplet merging, exit of the probe volume by the third axis or fail of the image processing on the first or second frame.

\subsection{Effect of the correcting factor}

The figure 9a presents the relative number of measured droplet in respect to the droplet diameter with both techniques. Only the F/M case is showed since the six cases presented similar trends. The results of the imaging technique are presented before and after the correction of the sampling inhomogeneity detailed in the section $\$ 2.4$. The correction decreases the peak located around 


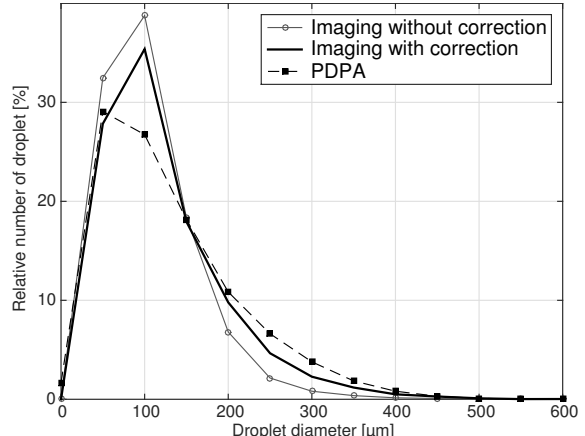

(a) Relative number

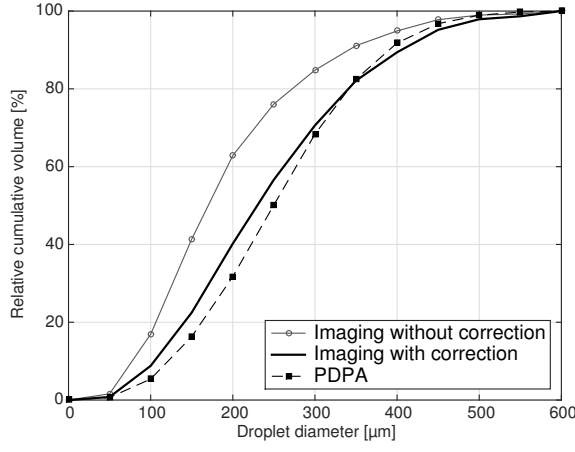

(b) Cumulative volume

Figure 9: Relative number of droplet and relative cumulative volume in respect to the droplet diameter for both techniques for the $\mathrm{F} / \mathrm{M}$ case. The imaging results are given before and after the correction of the sampling inhomogeneity.

$75 \mu \mathrm{m}$ and increases the proportion of droplets larger than $150 \mu \mathrm{m}$. The figure $9 \mathrm{~b}$ shows the relative cumulative volume in respect to the droplet diameter. On the correction the imaging results are closer to the PDPA data. An example of the relative variation of each component of the correcting factor according to the droplet diameter is presented on the figure 10. The depth of field and the correction of the field of view have a low range of variation equal to 1.54 and the preponderance of the velocity in the value of the correction factor. Since the velocity is increasing with the diameter, the correction is increasing the relative proportion of large droplets.

\subsection{Droplet size distribution}

Figure 11 compares the cumulative volumetric droplet size distribution between the PDPA and the imaging technique for the 6 reference nozzle-pressure combinations. Concerning the imaging technique, the 6 droplet size distributions are well differentiated showing that the imaging technique is able to measure small and coarse droplets with the same set-up. The smallest measured 


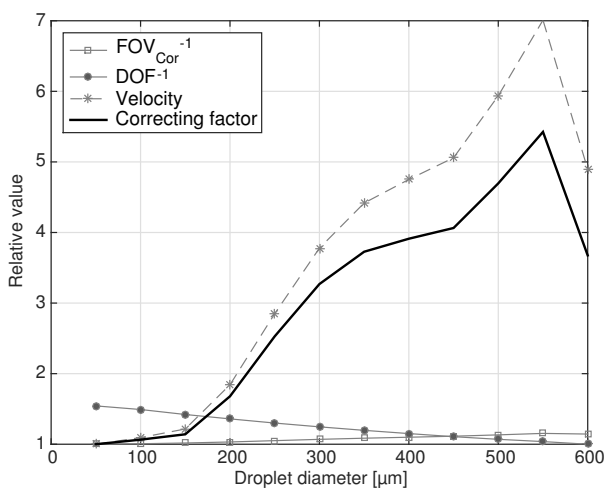

Figure 10: Relative value of the different correcting factors in respect to the droplet diameter for the $\mathrm{F} / \mathrm{M}$ case.

$1300 \mu \mathrm{m}$. The coarser sprays present less smooth curves because of the lower number of droplets recorded. The comparison between imaging technique and PDPA measurement provides some general trends. For the finest sprays $(V F / F$ $F / M)$, both techniques measured similar droplet size distributions. Whilst for the coarser sprays, there is a significative difference: for the $M / C$ and $C / V C$ sprays, the imaging technique measured a finer droplet size distribution and for the $V C / X C$ and $X C / U C$ sprays the imaging technique measured coarser droplet size distribution.

The Figure 12 compares the measurements of $D v_{10}, D v_{50}$ and $D v_{90}$ obtained with both techniques. $D v_{10}, D v_{50}$ and $D v_{90}$ are corresponding to the maximum particle diameter below which $10 \%, 50 \%$ and $90 \%$ of the sample volume exists, respectively. For all the sprays, the imaging technique gave a lower $D v_{10}$ than the PDPA. The difference between both measurements is roughly increasing with the droplet size spectrum. This observation is surprising since the imaging technique does not take into account the droplets smaller than $40 \mu \mathrm{m}$, so it was expected to overestimate the $D v_{10}$. The $D v_{50}$ measurements were quite similar between both techniques except for the $M / C$ case for which the difference is significant $(62 \mu \mathrm{m})$. The error bars show the standard error on the average. This error has been computed by considering the three scans as independent. The error is low for the most of the cases, except for the $D v_{90}$ measurements 


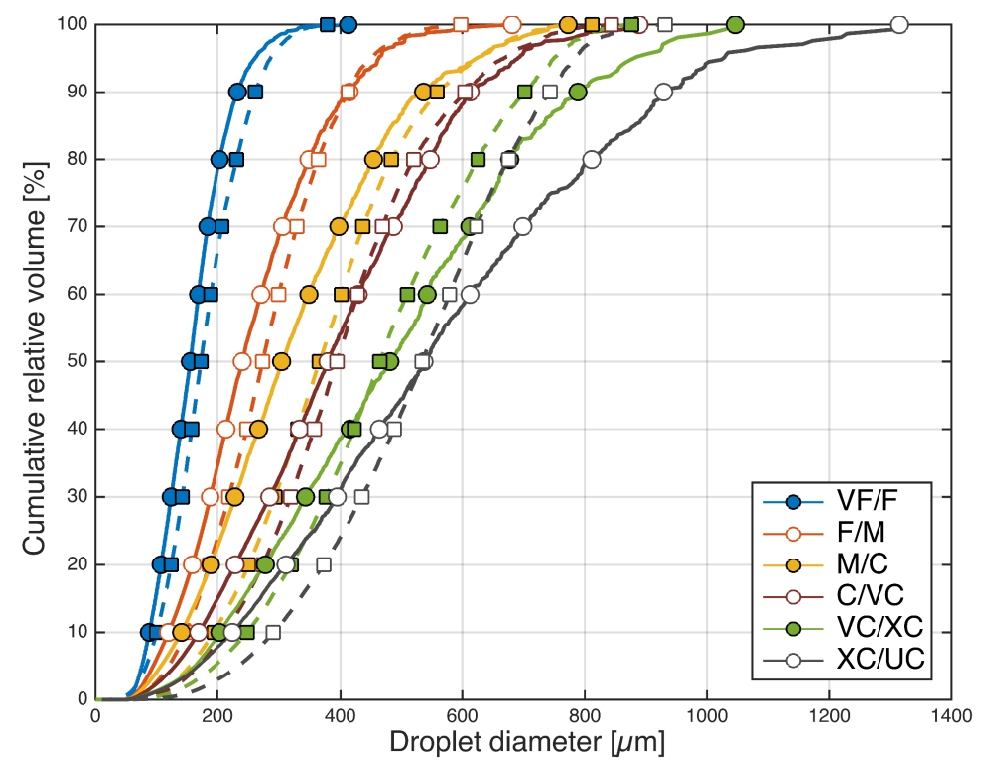

Figure 11: Cumulative volumetric droplet size distribution for the 6 spray class boundaries. Imaging technique and PDPA are represented by the circle with full lines and the square with the dashed lines respectively.

of the coarse nozzle with the imaging technique. This relatively high error can be explained by the low amount of large droplet recorded because of their lower presence and their lower sampling probability with the imaging technique. The $D v_{90}$ comparison shows large discrepancies for the coarser sprays $(V C / U C$ and $U C / X C)$. In these coarse sprays, the imaging technique recorded large $(\geq$ $1 \mathrm{~mm}$ ) and fast $(\geq 13 \mathrm{~m} / \mathrm{s})$ droplets which have a significant contribution on the final volumetric droplet size distribution. These droplets may not have been detected by the PDPA system because their diameter exceeded the maximum detectable diameter or because of their non sphericity. Table 3 presents the average and the standard deviation of the difference in term of $D v_{10}, D v_{50}$ and $D v_{90}$ between two neighboring spray classes i.e. $\Delta D v_{10 i}=\left(D v_{10 i+1}-D v_{10 i}\right)$. Imaging technique presents a higher spacing uniformity between each reference spray.

Table 4 presents for each technique the measured relative span factor (RSF) computed as: $R S F=\left(D v_{90}-D v_{10}\right) / D v_{50}$. For most of the sprays, the imaging 


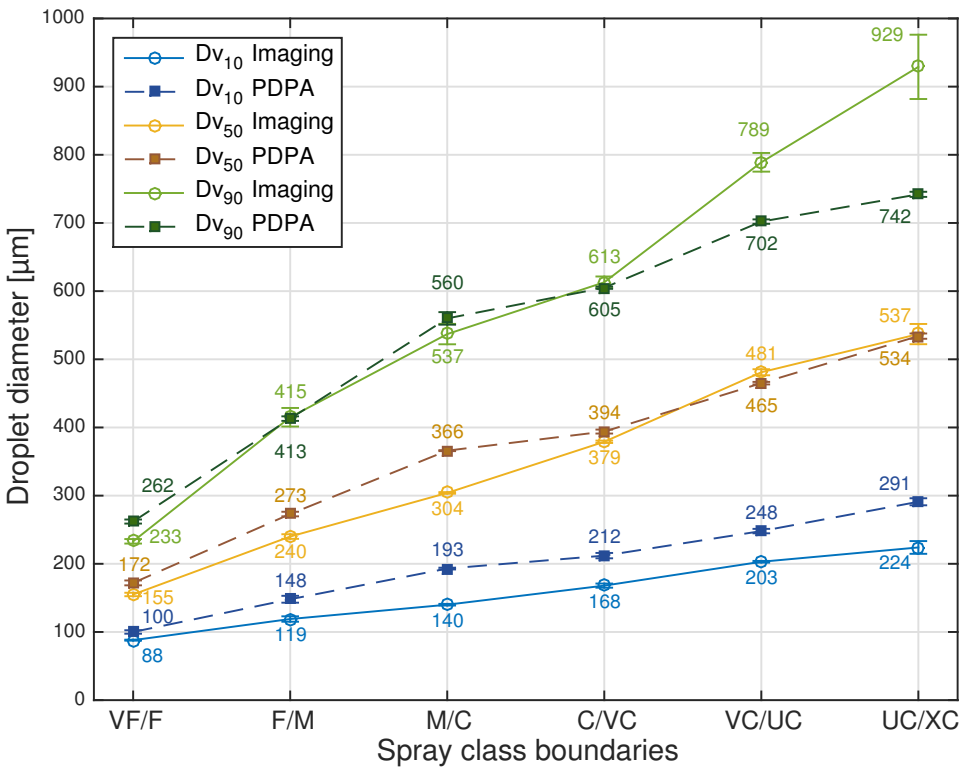

Figure 12: $D v_{10}, D v_{50}$ and $D v_{90}[\mu m]$ for the 6 reference sprays. Imaging technique and PDPA are represent by full lines with circles and the dashed lines with squares respectively.

Table 3: Average, standard deviation and coefficient of variation of the difference between following spray class boundary.

\begin{tabular}{|c|c|c|c|c|c|c|c|c|c|}
\hline & \multicolumn{3}{|c|}{$\Delta D v_{10}$} & \multicolumn{3}{|c|}{$\Delta D v_{50}$} & \multicolumn{3}{|c|}{$\Delta D v_{90}$} \\
\hline & Avg & Std & $\mathrm{CV}$ & Avg & Std & $\mathrm{CV}$ & Avg & Std & $\mathrm{CV}$ \\
\hline & {$[\mu m]$} & {$[\mu m]$} & {$[\%]$} & {$[\mu m]$} & {$[\mu m]$} & {$[\%]$} & {$[\mu m]$} & {$[\mu m]$} & {$[\%]$} \\
\hline Imaging & 27.3 & 6.1 & 22.3 & 76.5 & 18.1 & 23.7 & 139.6 & 43.1 & 30.1 \\
\hline PDPA & 38.2 & 12.2 & 31.9 & 72.4 & 28.4 & 39.2 & 96.0 & 53.3 & 55.5 \\
\hline
\end{tabular}


Table 4: Relative span factor measured for each spray class boundaries for PDPA and imaging technique.

\begin{tabular}{c|c|c|c|c|c|c} 
& $\boldsymbol{V} \boldsymbol{F} / \boldsymbol{F}$ & $\boldsymbol{F} / \boldsymbol{M}$ & $\boldsymbol{M} / \boldsymbol{C}$ & $\boldsymbol{C} / \boldsymbol{V} \boldsymbol{V}$ & $\boldsymbol{V} / \boldsymbol{U} \boldsymbol{C}$ & $\boldsymbol{U} \boldsymbol{C} \boldsymbol{X} \boldsymbol{C}$ \\
\hline \hline Imaging & 0.94 & 1.22 & 1.31 & 1.18 & 1.22 & 1.31 \\
PDPA & 0.94 & 0.97 & 1.00 & 1.00 & 0.98 & 0.85
\end{tabular}

technique presents a larger value of RSF with RSF values of 1.2 to 1.3 whilst PDPA measured almost constant RSF ranging around 1. For the coarsest spray, PDPA measured a surprisingly low value of RSF which may be explained by the difficulty of the PDPA to measure coarse droplet leading to an underestimation of the $D v_{90}$.

\subsection{Droplet velocity distribution}

In the present section, the comparisons are realized for the droplet vertical velocity since it's the only velocity component measured by the PDPA. The figure 13 shows the average velocity measured according to the droplet diameter class for both techniques. The average velocity has been computed with diameter classes of $50 \mu \mathrm{m}$ for bins having at least 25 droplets. Discrepancies between both techniques mainly appears for larger droplets. The source of these differences may be a combination of an error on size and velocity measurement, a too small sample or a difference in the operating conditions.

The figure 14 shows the cumulative volumetric droplet velocity distribution for the 6 different nozzle-pressure combinations. The droplet velocity is ranging from 0 to $20 \mathrm{~m} / \mathrm{s}$. For the finer sprays, the velocity is increasing with the droplet size. The highest speeds were found for the $\mathrm{C} / \mathrm{VC}$ nozzle-pressure combination. This high speed may justify the relatively higher percentage of rejected droplets observed for this nozzle-pressure combination with the imaging technique. The differences observed between both techniques mainly arise from the difference in term of droplet size measurements since the size/velocity behavior is similar with both technique. 

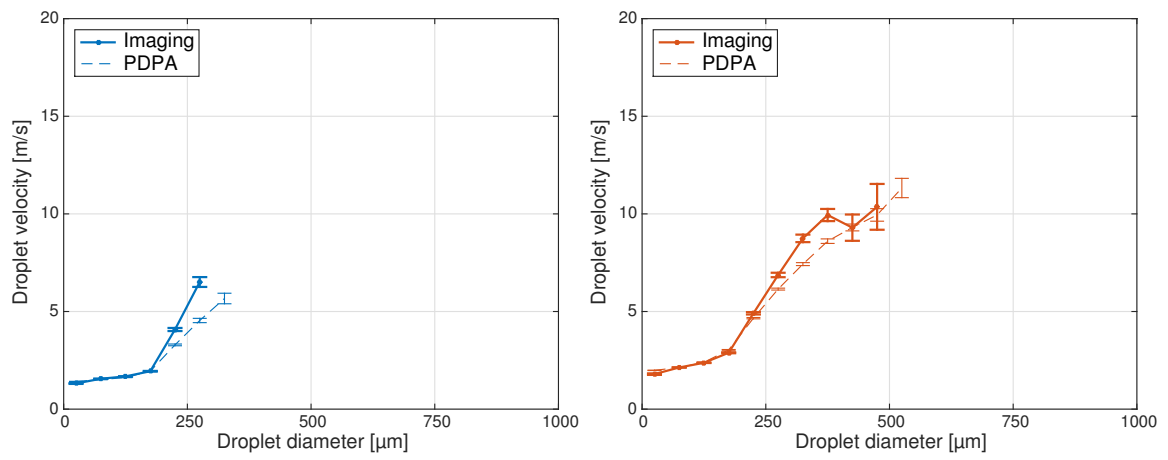

(a) $\mathrm{VF} / \mathrm{F}$

(b) $\mathrm{F} / \mathrm{M}$
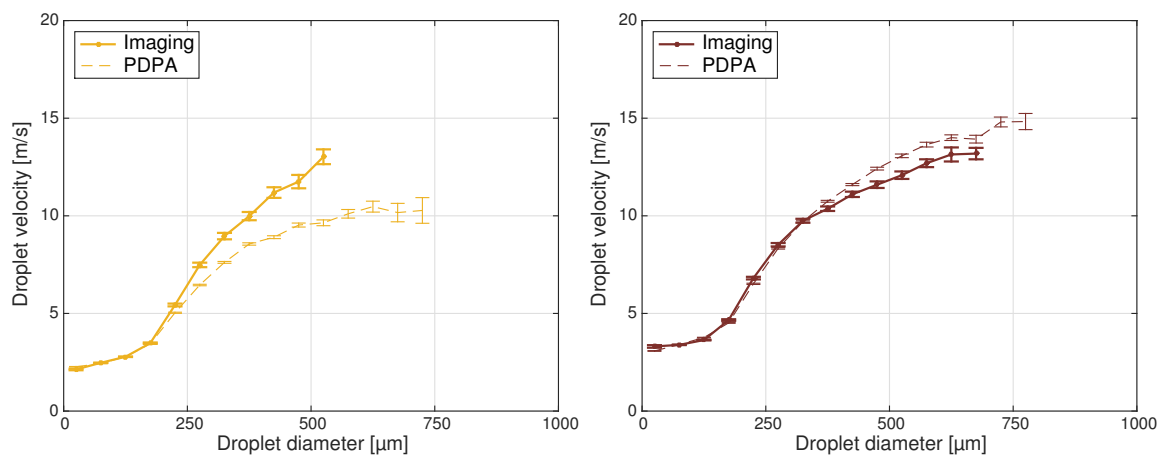

(c) $\mathrm{M} / \mathrm{C}$

(d) $\mathrm{C} / \mathrm{VC}$

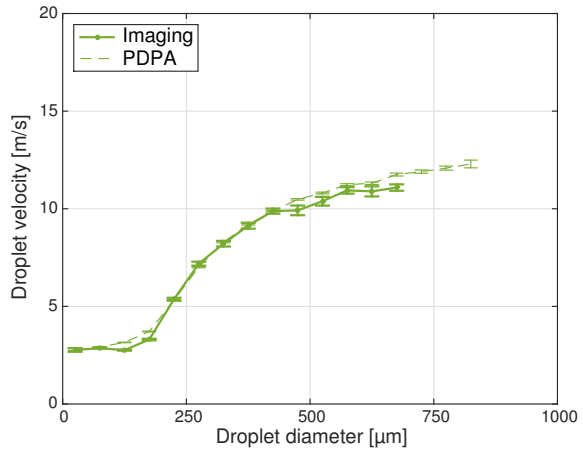

(e) $\mathrm{VC} / \mathrm{UC}$

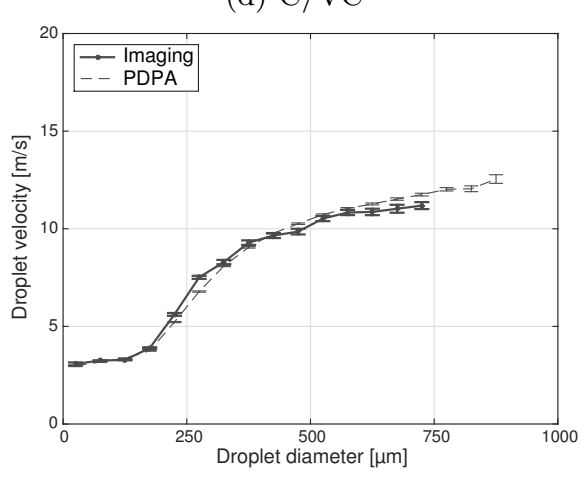

(f) $\mathrm{UC} / \mathrm{XC}$

Figure 13: Average velocity measured per diameter class of $50 \mu m$ with both techniques. The error bars indicate the standard error on the mean. 


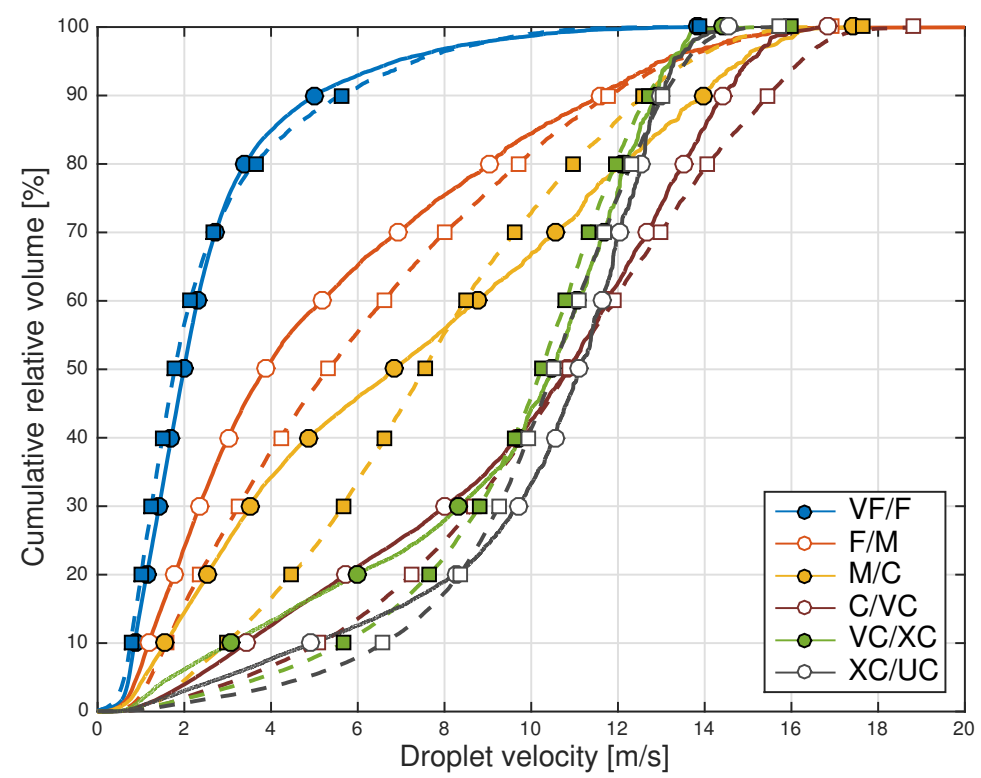

Figure 14: Cumulative volumetric droplet velocity distribution for the 6 different nozzlepressure combinations. Imaging technique and PDPA are represented by the circle with full lines and the square with the dashed lines respectively.

\section{Conclusion}

A digital image acquisition technique and analysis algorithm was proposed for droplet size and velocimetry measurements. The image acquisition set-up and the image processing method has been detailed. The droplet size distributions of a set of reference sprays defined in the ISO/DIS 25358 were measured using the proposed imaging technique and a PDPA laser. Concerning the imaging technique, the 6 sprays droplet size distributions were differentiated well. The smallest droplet measured had a diameter of $40 \mu \mathrm{m}$ and the largest droplet measured had a diameter of $1300 \mu \mathrm{m}$. The comparison between imaging technique and PDPA measurement provided some global trends. For the finest spray $(V F / F)$, both techniques measured a similar droplet size distributions. Whilst

${ }_{435}$ for the coarser sprays, there is a significant difference: for the $F / M$ and $M / C$ sprays, the imaging measured a finer droplet size distribution and for the $C / V C$, $V C / X C$ and $X C / U C$ sprays the imaging technique measured coarser droplet 
size distribution. PDPA measurements tend to measure an equivalent $D v_{50}$, a higher $D v_{10}$ and a lower $D v_{90}$ than the imaging technique leading therefore to a lower relative span factor. Velocity measurements showed good agreement between both techniques except for one nozzle/pressure combination. Therefore, comparison of two measurements realized with each method should be realized carefully knowing these differences. The $D v_{50}$ seems to be the best parameter for comparisons since both techniques provide similar value.

\section{Aknowledgments}

This work was supported by the Fonds de la Recherche Scientifique - FNRS under the FRIA grant $n^{\circ} 97364$.

\section{References}

Canny, J., 1986. A computational approach to edge detection. IEEE Transactions on Pattern Analysis and Machine Intelligence 8, 679-698.

Cousin, J., Berlemont, A., Ménard, T., Grout, S., 2012. Primary breakup simulation of a liquid jet discharged by a low-pressure compound nozzle. Computers \& Fluids 63, 165-173.

Damaschke, N., Gouesbet, G., Gréhan, G., Mignon, H., Tropea, C., 1998. Response of phase doppler anemometer systems to nonspherical droplets. Applied optics $37,1752-1761$.

Fritz, B.K., Hoffmann, W.C., Czaczyk, Z., Bagley, W., Kruger, G., Henry, R., 2012. Measurement and classification methods using the ASAE S572.1 reference nozzles. Journal of Plant Protection Research 52, 447-457.

Hewitt, A.J., Valcore, D.L., Teske, M.E., Schick, R.J., 1998. Drop size classifications for agricultural sprays, in: Proceedings of the 11th Annual conference on liquid atomization and spray systems, May 1998, Sacramento, CA, USA. 
Holterman, H.J., van de Zande, J.C., Porskamp, H.A.J., Huijsmans, J.F.M., 1997. Modelling spray drift from boom sprayers. Computers and Electronics in Agriculture 19, 1-22.

Kang, L., Guo, L., Gu, Z., Liu, D., 2008. Wind tunnel experimental investigation of sand velocity in aeolian sand transport. Geomorphology 97, 438-450.

Kashdan, J., Shrimpton, J., Whybrew, A., 2003. Two-phase flow characterization by automated digital image analysis. part 1: Fundamental principles and calibration of the technique. Particle \& Particle Systems Characterization 20, $387-397$.

Kwak, B.M., Lee, J.E., Ahn, J.H., Jeon, T.H., 2009. Laser diffraction particle sizing by wet dispersion method for spray-dried infant formula. Journal of Food Engineering 92, 324-330.

${ }_{475}$ Lebeau, F., Verstraete, A., Stainier, C., Destain, M.F., 2011. RTDrift: A real time model for estimating spray drift from ground applications. Computers and Electronics in Agriculture 77, 161-174.

Lecuona, A., Sosa, P.A., Rodrguez, P.A., Zequeira, R.I., 2000. Volumetric characterization of dispersed two-phase flows by digital image analysis. Measurement Science and Technology 11, 1152-1161.

Lefebvre, A., 1988. Atomization and Sprays. Taylor \& Francis.

Li, T., Nishida, K., Hiroyasu, H., 2011. Droplet size distribution and evaporation characteristics of fuel spray by a swirl type atomizer. Fuel 90, 2367-2376.

Liu, X., Doub, W.H., Guo, C., 2010. Evaluation of droplet velocity and size from nasal spray devices using phase Doppler anemometry (PDA). International Journal of Pharmaceutics 388, 82-87.

Massinon, M., Boukhalfa, H., Lebeau, F., 2014. The effect of surface orientation on spray retention. Precision agriculture 15, 241-254. 
Massinon, M., Dumont, B., De Cock, N., Salah, S.O.T., Lebeau, F., 2015. Study of retention variability on an early growth stage herbaceous plant using a 3D virtual spraying model. Crop Protection 78, 63-71.

Massinon, M., Lebeau, F., 2012. Experimental method for the assessment of agricultural spray retention based on high-speed imaging of drop impact on a synthetic superhydrophobic surface. Biosystems Engineering 112, 56-64.

Nuyttens, D., Baetens, K., De Schampheleire, M., Sonck, B., 2007. Effect of nozzle type, size and pressure on spray droplet characteristics. Biosystems Engineering 97, 333-345.

Rein, M., 1993. Phenomena of liquid drop impact on solid and liquid surfaces. Fluid Dynamics Research 12, 61-93.

Sirignano, W., Mehring, C., 2000. Review of theory of distortion and disintegration of liquid streams. Progress in Energy and Combustion Science 26, 609-655.

Snyder, H.E., Senser, D.W., Lefebvre, A.H., Coutinho, R.S., 1989. Drop size measurements in electrostatic paint sprays. Industry Applications, IEEE Transactions on 25, 720-727.

Southcombe, E.S.E., Miller, P.H.C., Ganzelmeier, H., van de Zande, J.C., Miralles, A., Hewitt, A.J., 1998. The International (BCPC) spray classification system including a drift potential factor, in: Proceedings of the Brighton Crop Protection Conference-Weeds.

Teske, M.E., Bird, S.L., Esterley, D.M., Curbishley, T.B., Ray, S.L., Perry, S.G., 2002. Agdrift@: A model for estimating near-field spray drift from aerial applications. Environmental toxicology and chemistry 21, 659-671.

Walklate, P.J., 1987. A random-walk model for dispersion of heavy particles in turbulent air flow. Boundary-Layer Meteorology 39, 175-190. 
${ }_{515}$ Wang, Y., Miller, D.R., Anderson, D.E., McManus, M.L., 1995. A lagrangian stochastic model for aerial spray transport above an oak forest. Agricultural and Forest Meteorology 76, 277-291.

Widmann, J.F., 2001. Phase doppler interferometry measurements in water sprays produced by residential fire sprinklers. Fire Safety Journal 36, 545567.

Womac, A.R., 2000. Quality control of standardized reference spray nozzles. Transactions of the ASAE 43, 47-56.

Zabkiewicz, J.A., 2007. Spray formulation efficacy - holistic and futuristic perspectives. Crop Protection 26, 312-319.

Zhou, X., D'Aniello, S.P., Yu, H.Z., 2012. Spray characterization measurements of a pendent fire sprinkler. Fire Safety Journal 54, 36-48. 